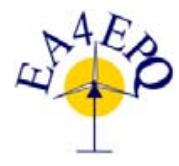

International Conference on Renewable Energies and Power Quality (ICREPQ'16)

Madrid (Spain), $4^{\text {th }}$ to $6^{\text {th }}$ May, 2016

Renewable Energy and Power Quality. Fournal (RE\&PQJ)

ISSN 2172-038 X, No.14 May 2016

\title{
A Study of the Induction Motor/ASD Immunity in the Presence of Voltage Sags
}

\author{
Juan Carlos Gómez \\ Electrical Power System Protection Institute \\ Rio Cuarto National University \\ Rio Cuarto, Argentina. \\ jcgomez@ing.unrc.edu.ar \\ (Correspondent author)
}

\author{
Medhat M. Morcos \\ Electrical and Computing Engineering Department \\ Kansas State University \\ Manhattan, Kansas, USA.
}

\begin{abstract}
Adjustable speed drives (ASDs) are sensitive to power quality problems, especially voltage sags. The effect of the magnitude and duration of voltage sags on ASD behavior is related to the energy deficit. On the other hand, the induction motor operation is sensitive to voltage sags in regard to the restart possibility as well as the drop in the motor speed. ASD and induction motor immunities need to be coordinated, being presented the required methodology. A complete study of the involved equipment immunities is needed in order to obtain a better behavior in the presence of voltage sags.
\end{abstract}

Index Terms-ASDs, Power Quality, Energy deficit, Speed drop, Voltage sags.

\section{I- INTRODUCTION}

Voltage sag or also called voltage dip, is one of the most important power quality disturbances; it is responsible for more than $75 \%$ of customer's complaints due to short-time power quality events [1].

Adjustable speed drives (ASDs) are widely used in the industry, being several of them connected jointly in a production line, line that is frequently detained if anyone of such equipment is out of service. They are particularly sensitive to power quality problems, especially voltage sags $[2,3]$. This is the cause of their high incidence in industrial production line detentions and of the importance for their detailed study need. The effect of the magnitude and duration of voltage sags on ASD behavior is related to the energy deficit [4].
On the other hand, the induction motor operation is sensitive to voltage sags in regard to the re-start possibility as well as the drop in the motor speed. Both effects depend on the motor mechanical load and the voltage recovery [5]. The first aspect depends on the torque required for the reacceleration against the counter (opposing) torque. The behavior of the induction motor is explained in the presence of voltage sags, considering the energy supplied when operating as an induction generator [6].

It is also important to take into account the effect of the remaining connected loads that are in the same restart process, where the frequently delayed schemes should be applied in the restart of non-essential loads.

\section{II- ASD IMMUNITY}

In simplified form, as it is shown in Fig 1, the circuital scheme of an ASD can be considered as three stages, blocks or black boxes that from the supply toward the motor are respectively "rectification" (controlled or not), "DC link", and "inverter". The ASD can be damaged in the event of being presented a voltage sag, suffering of high overcurrents that are also accompanied by motor torque oscillations with their rising risk of mechanic damage.

When the study of the behavior of these stages is carried out, it should be considered their protection especially against overcurrents, undervoltage and underspeed. Such protection are usually manufacturer-adjusted (before leaving factory), being possibly selected with excessively conservative ideas, for what is feasible in order to reduce their high sensibility 
(previous consultation to the maker) to reduce the level of the undervoltage limit (usually fixed between the 70 and 90\%) and of the trip overcurrent (usually adjusted between the 120 and $170 \%$ of the rated current), with what will diminish the number of the ASD out of services.

It can be affirmed, that the Sensitive Equipment (SE) in general and the ASD in particular are highly sensitive to the voltage sags deep, duration, and unbalance [2].

As it was already mentioned, the ASD out of service is determined by the energy deficit, in such a way that is necessary to analyze where energy can be stored that allows to overcome this transient deficit, either in the ASD like in the motor and in the propelled load. In the ASD energy can be stored in the capacitor of the intermediate block or DC link, action that today is frequently adopted [1].

Here only will be studied the necessary and indispensable facts about the ASD operation in order to understand the effect of the voltage sags, without deepening on other ASD particular operation characteristics.

The first stage of the typical scheme of the ASD is the three-phase rectifier bridge that can be controlled or not, possessing or not in the first case the possibility to act as inverter and to carry out regenerative or of energy saving tasks. The second stage poses capacitors and inductors, in order to obtain a filtered and of low ripple DC output, that is usually denominated as DC link. The last stage is frequently the corresponding to the inverter that converts the DC voltage again in AC voltage, of variable amplitude and frequency, generally using the PWM methodology, being in some cases able to reverse the energy flow direction from the motor toward the source.

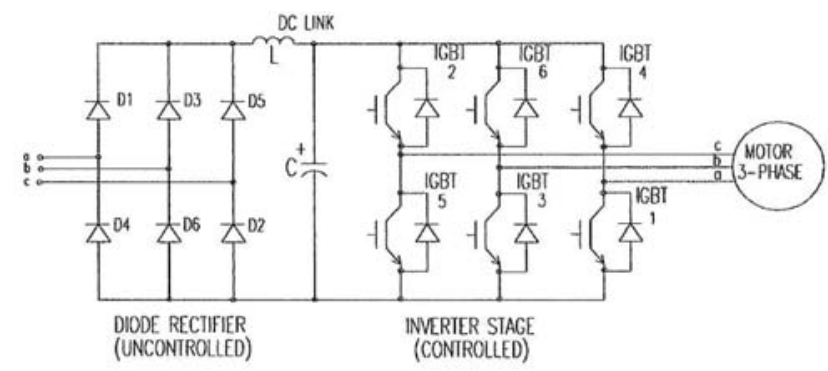

Fig. 1 Typical schematic circuit of an AC ASD.

In the event of using diodes in the rectification (not controlled), the current flow is blocked until the voltage on the capacitor terminals is inferior to that of the supply. In presence of a voltage sag, the capacitor supplies the stored energy to the load, being exhausted in a few cycles, in front of that the motor reduces its speed depending on the voltage sag depth, of its mechanical load and of its inertia. Something of residual voltage is maintained in terminals of the motor, for magnetic storage (remnant), so that in the moment that the supply voltage recovers, if there are not special strategies of mitigation, the overcurrent that is presented possesses very high values, reaching to 10 times the rated current. In that moment the voltage of the ASD and that of the motor cannot be synchronized and it can happen that the ASD maintains its regulation previous to the perturbation that is to say with the same modulation index and the same statoric pulsation [7].

An overcurrent phenomenon, similar to the one explained in simplified form, it is presented in the event of having unbalanced supply (either for the presence of a non-threephase voltage sag or due to unbalanced steady state supply).

In steady state régime, when possessing the motor usually only three conductors, the three-phase full-wave rectifier detects the line-to-line voltage and absorbs of the grid a nonsinusoidal current with two positive and two negatives crests for cycle, the typical waveform denominated "rabbit ears". This waveform is caused by the capacitor that absorbs current as the energy is transferred toward the motor, only governed by the voltage difference between its terminals and the one supplied from the source. If there is imbalance, it can happen that the voltage that is applied on the capacitor terminals be inferior to the remainder for what does not absorb current in that moment, but when passing to the following phase, the voltage it is overcome and in a big amount since the time of energy delivery of the capacitor to the load was abnormally long, what originates a high overcurrent that can damage the first stage (inverter or single rectifier) or to produce the trip of the overcurrent protection [8]. A similar effect is presented in the event of existing a phase jump. Fig 2 shows the wave shapes for normal conditions and in presence of the cited perturbation.

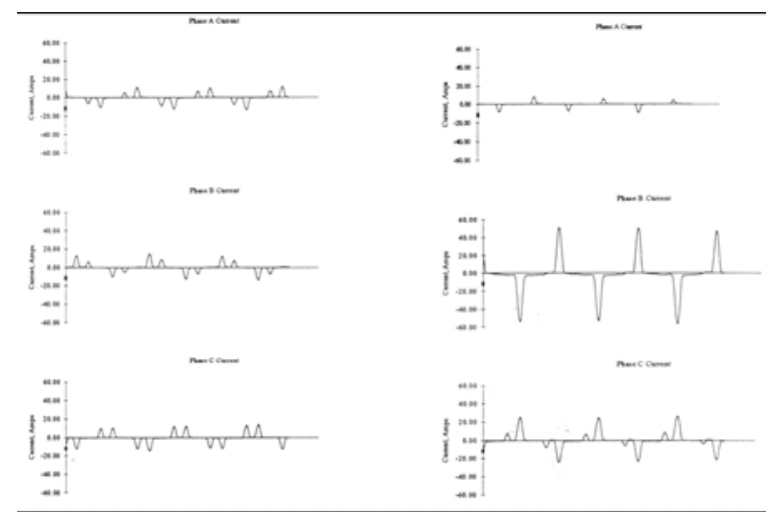

Fig 2 ASD grid absorbed current under balanced and unbalanced conditions [9].

When the first stage uses controlled rectifier (SCR), in presence of the voltage drop, the feedback detects it and tries to maintain the block output voltage for what reduces the thyristor trip angle. When the system voltage is recovered, the SCRs trip angle will be saturated and not synchronized with the voltage of the motor emf neither with the grid frequency, for what important overcurrent appears that can trip the equipment protection [8]. 


\section{III- INDUCTION MOTOR BEHAVIOUR}

The behavior of the induction motor in presence of a voltage sag depends on its load state and of the depth of the sag. To understand the behavior it should be clearly understood the interaction between the voltage applied to the motor terminals and the emf developed by the same one. The difference among these two magnitudes is which determines the power input or output to the machine. Under steady state, if the applied voltage overcomes the emf, the machine is operating as motor, contrary case is a generator supplying active power to the grid. Under transient conditions the behavior is similar; if the voltage sag is of depth just as so that the emf prevails, the equipment behaves as a generator supplying energy to the grid while it maintains its excitation for remnant magnetism. The oscillogram of fig 3 shows the current of an induction motor when a deep voltage sag takes place, where the initial current contribution can be seen, which is quickly vanished when the remnant magnetism is exhausted in a few cycles, with a light speed reduction. When the cause of the voltage sag disappears, the motor restart takes place with an important overcurrent that is regularized at one time of the order of the corresponding to the full voltage motor start [10].

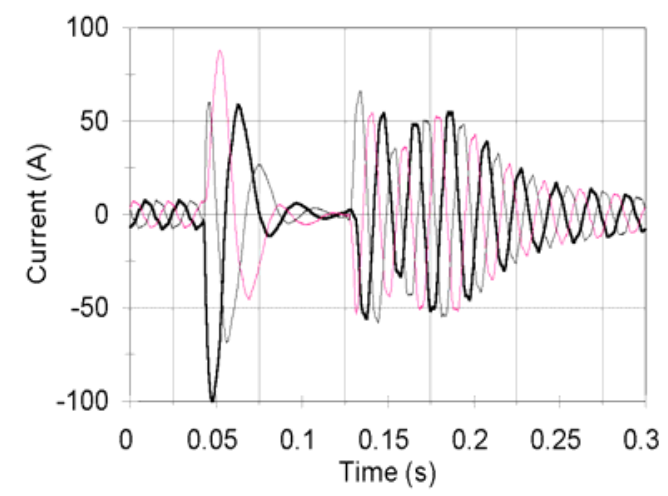

Fig. 3 Induction motor current and the delayed re-start in the presence of a deep voltage sag.

In order to analyze the induction motor wanted behavior in presence of a voltage sag, firstly the premises related with the continuity of the productive process should be specified [1]:

- The motor should stay connected the longest possible time in order to reduce cost of production losses.

- It should be kept in mind the conditions, before, during, and after the voltage sag.

- It should not put in risk the motor integrity.

- The motor should not remain connected beyond the point in the one that the propelled load can recover the steady state conditions.

- It is not justifiable to extend the motor connection further on that the time supported by the remaining elements of the productive line.
In general, only the graph of the allowed speed-drop as function of the voltage-sag magnitude and duration is used. The graph is shown in Fig. 4 in its typical form. Information about equipment sensitivity to voltage sags is normally presented in graphical form, known as immunity curves; the most known are CBEMA, ITIC, and SEMI F47, shown in Fig. 5. The graphs are time/voltage characteristics (TVC) that indicate the border of undervoltage at normal operation as a function of time [5].

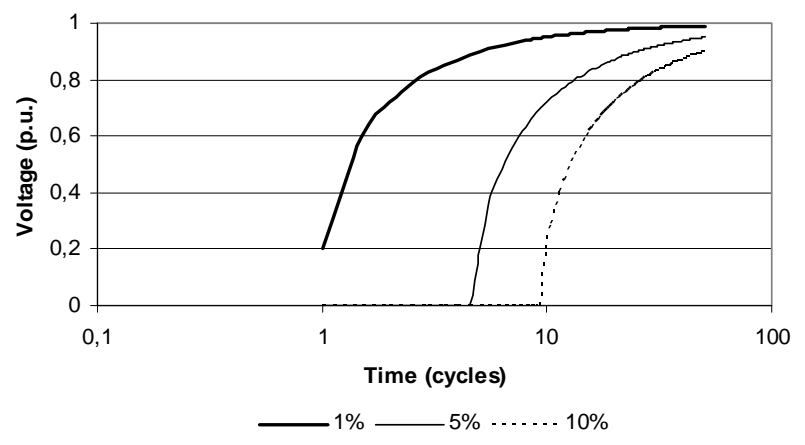

Fig. 4 Induction motor immunity in the presence of a voltage sag as function of the acceptable speed-drop.

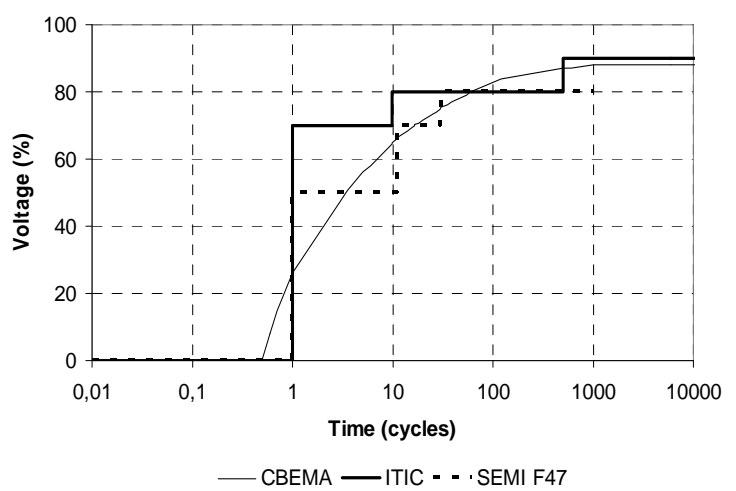

Fig. 5 CBEMA, ITIC, and SEMI F47 immunity curves [10].

The equivalent curve of motor based on their speed and torque, is given by the following simplified expression [5]:

$$
\Delta \mathrm{s}=\left(1-\mathrm{V}^{2}\right) /(2 \mathrm{H}) \Delta \mathrm{t}
$$

Where: $\Delta s=$ slip increment, $V=$ magnitude of the voltage sag, $\mathrm{H}=$ constant of inertia (energy stored in function of the rated power, $\mathrm{kW} . \mathrm{s} / \mathrm{kVA}), \Delta \mathrm{t}=$ duration.

The speed drop of the induction motor in presence of a reduction of the applied voltage can be analyzed using the concept of effects addition. The effects are the desacceleration for zero voltage more the acceleration with 
undervoltage (below the rated voltage) whose composition gives the speed variation shown in Fig 6 [11].

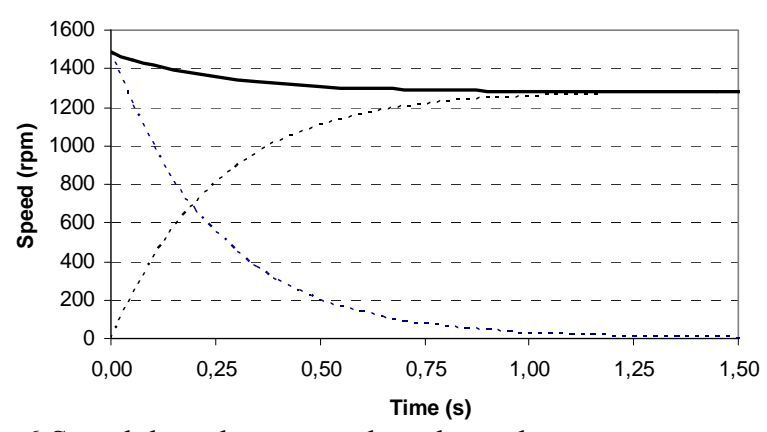

Fig 6 Speed drop due to supply voltage drop.

The speed change as time function is given by the following expression [6]:

$$
\omega(t)=\omega_{s} e^{-\tau 1} t+A\left(1-e^{-\tau 1} t\right)
$$

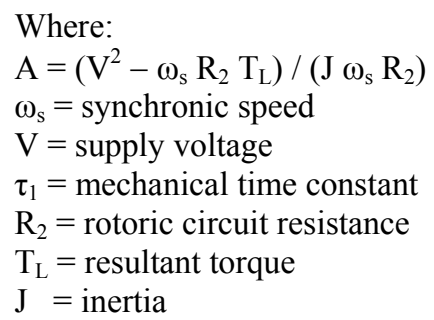

\section{IV- SPECIFIC-ENERGY CONCEPT}

As it was already mentioned, the capability of the sensitive equipment in general (without pieces in movement) to support a voltage sag without passing out of service, it is directly related with the energy stored in their DC link or DC block. The energy amount is approximately function of the squared voltage value. This is the energy that maintains working the device while the supply voltage is below the equipment needs.

Due to cost reasons, the maker of the sensitive equipment supply so low immunity as it is required by most of the customers, frequently the customer with higher requirements is in the necessity of adding auxiliary devices (to increase the size of the DC link capacitor or to add an UPS) in order to improve the sensitive equipment immunity.

The CBEMA curve of Fig 5 shows the existence of a very smooth correlation voltage-time that makes think of the existence of a simple function that represents it. On the other hand the curve ITIC is given in staggered form, equally that the SEMI F47, nevertheless on the average they follow a law like the corresponding to CBEMA (see Fig 5) [10].

The immunity of a sensitive equipment in presence of voltage sags is very affected by the voltage previous to the perturbation, which confirms the concept that the voltage above $87 \%$ is used for storage, being liberated later on in the event of a perturbation like a voltage sag. This $87 \%$ of the rated voltage is the minimum value given by the curve
CBEMA, for which the equipment will be able to work in indefinite form without presenting behavior limitations. This tension is defined as "inherent design voltage".

The concept of stored energy leads to the presence of a law of variation related with the multiplication of the squared voltage and the time, curve that is practically coincident with the CBEMA, the former obtained based on the relationship: $\mathrm{V}^{2} . \mathrm{t}=$ constant. For instance, the values of these parameters for the CBEMA curve are 4,400 $\%^{2}$ cycles and $87 \%$, respectively $[1,6]$.

The previously defined methodology (CBEMA-ITICSEMI F47 curves) agrees in considering as nominal value of the voltage sag to the maximum voltage drop. The difficulty is presented when it is wanted to assign the perturbation duration, since in the real world the voltage drop is not always a very abrupt (rectangular sag) neither its depth is constant, but rather it is function of the characteristics of the system and involved equipment.

Another of the difficulties in the voltage sag characterization roots in the presence of sags of progressive magnitude or that evolves of a fault type in another.

The application of the energy concept, leads to the change of the previous equation, transforming now in: $\int \mathrm{v}^{2} \mathrm{dt}=\mathrm{k}$.

Where $\int \mathrm{v}^{2} \mathrm{dt}$ receive the name of "specific energy" in similar form similar to the $\mathrm{I}^{2}$. $\mathrm{t}$, value that is widely used in overcurrent protection studies [6].

The introduction of this integral for each sensitive equipment solves the ambiguity problem, giving a complete characterization of the behavior in presence of voltage sags. The proposed methodology is able to consider the value of the initial step of the sag, which is usually presented or to the voltage development on the fault arc, effect that also takes place in systems with double feeding showing a sudden step when the first protection trips.

The capability of a three-phase equipment to withstand voltage sags is not the same when it is in presence of singlephase, double-phase or three-phase perturbation. In the case of a single-phase fault, the phases not involved in the perturbation generate an overvoltage surge that over-feeds the equipment, improving their immunity. It is not difficult to understand the concept of specific energy for the capability to withstand voltage sags of the three-phase sensitive equipment that should be an unique value to compare with the values of each phase of the sag, depending on the characteristics of the under study event (voltage sag). The validation of applicability of the concept of constant specific energy can be made based on results of available events in the literature, so much for individual equipment as for complete industrial processes [1].

\section{V- CONVOLUTING IMMUNITY CURVES}

In most of the industrial, commercial and some domestic applications, complex equipment is composed of several devices, each having its own immunity time/voltage characteristics. The operation should be satisfactory if each of the devices is operating properly, thus the resulting immunity 
curve is above and to the left of the convoluted curve, including all individual curves. In this way the immunity of an industrial production line, one medium voltage feeder, a shopping center, or a trade center, can be easily represented by the convoluted time/voltage characteristic curve, as shown in Fig. 7. While more voltage-sag SEs are included, the convoluted composed curve drifts to a uniform line similar to the CBEMA curve. The individual and convoluted TVC curves can be represented by applying the voltage specific energy concept $\left(\mathrm{V}^{2} \mathrm{t}=\right.$ constant $)$, which as was mentioned allows the representation of the complete device immunity by two parameters, the "specific energy" and the "inherent design voltage" $[6,13]$.

The study of the induction motor/ASD immunity is conducted using the CBEMA, ITIC or SEMI F47 curves, together with the analytical methodology presented elsewhere Fig.3 [1].

The analytical representation is based on applying the concept of the voltage specific-energy that allows the analysis of the whole equipment immunity through the convoluting (combining) of the individual equipment immunity curves of diverse equipment. Fig. 7 shows a hypothetical example of a chain of immunities with three links (sensitive equipment) together with the convoluting curve that represents the combined immunity. For the under study case, it should be included in the graph the ASD immunity curve (for the conditions adjusted in the protection), that of the motor in function of the allowed speed drop and the homologous of any other involved sensitive equipment [13].

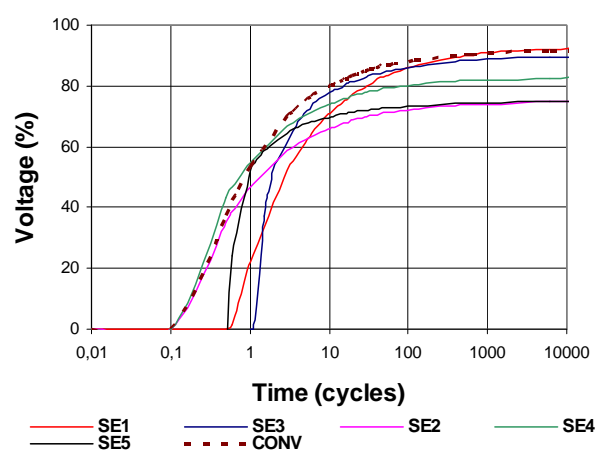

Fig. 7 Individual immunities and combined immunity curves.

An additional complication exists for the study here presented that is related with the remaining loads that are simultaneously restarting with the ASD-motor under analysis. These loads during the restart process absorb from the grid high current values that cause a significant system voltage drop, for what the under analysis equipment will have less available energy being able to originate a lengthening of the restart process and in extreme cases to impede it. Due to this difficulty, the remaining loads should be studied, proceeding to classify them based on their initial prescindibility, incorporating delays in order to allow the restart of the critical loads [12].

\section{VI- Mitigation MeAsures}

The mitigation measures are applicable to the ASD by means of the increment of capacitor size in the DC-link, and by the change of the operation strategy during the presence of voltage sags.

The mitigation strategies are [6]:

1. To compensate the fluctuation in the DC link, maintaining constant the supplied torque.

2. To wait before restarting the machine, allowing its the complete standstill.

3. Stop quickly to re-start under similar conditions to the normal start.

4. Restart after $300 \mathrm{~ms}$ to $2 \mathrm{~s}$, allowing the disappearance of the emf, being desirable to have a speed detector to restart with the frequency corresponding to the real speed.

5. Resynchronize the inverter with the voltage generated by the motor in that instant.

6. To maintain synchronized the motor, restarting immediately that the rated voltage is recovered, supplying kinetic energy from the motor to the capacitor to reinforce its falling charge.

7. To use DC feeding from general bus-bars for several ASDs, using the regenerated energy from other motors. It can be supplemented by means of the installation of storage equipment connected to the general bus-bar.

The possible solutions to improve the induction motor immunity from the viewpoint of the mechanical load - or shaft torque - are: (the explained strategies are only based on "intelligence" and not in auxiliary sources).

The application of these strategies leads to reductions of speed of bigger or smaller measure according to the load state, supposing that these speed drops are acceptable. In case the production process does not withstand these speed drops, it will be appealed to the employment of energy storage devices, as additional capacitors, batteries, superconductor coils, flywheels, UPSs, etc. whose analysis is similar for applications in individual sensitive equipment.

From an extensive investigation work published recently, the following conclusions of interest can be extracted: [14] - All the immunity curves present the same form, a vertical part, one elbow, and followed by a horizontal portion.

- The immunity in general is increased as the regulation of work speed diminishes.

- The start angle of the voltage sag and the phase jump do not affect to the equipment immunity.

- The voltage previous to the beginning of the perturbation influences the immunity. Higher voltages reduce the susceptibility of pass to out-of-service and the reverse.

- If the load works to constant torque, the sensibility increases as the load increases, for instance for $100 \%$ in torque the elbow coordinates are $15 \mathrm{~ms}$ and $71 \%$, passing to $175 \mathrm{~ms}$ and $60 \%$ for zero torque (no-load).

- The number of phases involved in the voltage sag affects the sensibility, being the most immune the case of single-phase 
fault, being followed by the two-phase, ending with the threephase fault being the most sensitive. For one equipment the variation of the coordinates of the elbow goes from $160 \mathrm{~ms}$, $60 \%$ up to $15 \mathrm{~ms}, 72 \%$ respectively.

\section{VII- CONCLUSIONS}

It is concluded that a complete study of the involved equipment immunities is needed in order to obtain a better behavior in the presence of voltage sags.

\section{REFERENCES}

[1] J. C. Gómez, Calidad de potencia: para usuarios y empresas eléctricas, Editorial EDIGAR S.A., ISBN: 987-97785-2-9, 2005.

[2] R.C. Dugan, M. McGranaghan, H.W. Beaty. Electrical Power Systems Quality. McGraw-Hill, New York, 1996.

[3] IEEE Standard 1346. IEEE Recommended Practice for Evaluating Electric Power System Compatibility with electronic process equipment. 1998

[4] S. Djokic et al Sensitivity of personal computers to voltage sags and short interruptions, IEEE Transactions on Power Delivery, Vol. 20, pp. 375-383, 2005.

[5] M.H. Bollen, Understanding Power Quality Problems, Voltage Sags and Interruptions. IEEE Press, USA, 2000

[6] J.C. Gómez, M.M. Morcos, Power Quality: Mitigation Technologies in a Distributed Environment, Chapter 10. Springer, London, 2007.
[7] A. Davis, J. Maire, M. Dessoude, Influence of voltage dips and sags characteristics on electric machines and drives: evaluation and perspective, Power Quality Assurance Conference, II, 1994.

[8] E. Collins, A. Mansoor, Effects of voltage sags on AC motor drives, IEEE 1997Annual textile, fiber and film industry conference, Greenville, SC, USA, May, pp. 68, 1997.

[9] J. L. Durán-Gómez, P. Enjeti, A low cost approach to improve the performanceof an adjustable speed drive (ASD) under voltage sags and short-term power interruptions, IEEE Applied Power Electronics Conference, New York, USA, February, pp.587-591, 1998.

[10] J.C. Gomez and M.M. Morcos, Specific energy concept applied to the voltage sag ride-through capability of sensitive equipment in DGembedded systems, IEEE Transactions on Power Delivery, vol. 18, no. 3, pp. 1590-1591, 2003.

[11] J.C. Gómez, M.M. Morcos, Coordination analysis of voltage sag and over-current protection in electrical systems with distributed generation, IEEE Transactions on Power Delivery, Vol. 20, pp. 214 $218,2005$.

[12] J.C. Gómez, M.M. Morcos, Voltage sag and recovery time in repetitive events, IEEE Transactions on Power Delivery, Vol. 17, pp. 1037-1043, 2002.

[13] J. C. Gómez, M. M. Morcos, Specific Energy Concept Applied to Electronic Equipment in Industrial Processes Having Distributed Generation, ICREPQ'12, March 28 - 30, Santiago de Compostella, Spain, 2012

[14] S. Djokic et al, Sensitivity of AC adjustable speed drives to voltage sags and short interruptions, IEEE Transactions on Power Delivery, Vol. 20, $\mathrm{N}^{\circ}$ 1, January, pp. 494-505, 2005. 